\title{
La verticalización como régimen urbano. El caso de las ciudades chilenas*
}

Fecha de recepción: 22 de marzo de 2018 । Fecha de aprobación: 24 de agosto de 2019 । Fecha de publicación: 29 de mayo de 2020

Jorge E. Vergara Vidal

Universidad de Valparaíso, Chile

ORCID: 0000-0002-7712-4090 $\quad$ jorge.vergaravi@uv.cl

Resumen La proliferación de las edificaciones de altura para uso residencial en los paños centrales y pericentrales de las ciudades chilenas es un fenómeno reciente, cuya intensidad alienta la pregunta sobre sus efectos en la interacción urbana. El presente texto explora los registros y relatos que dan pie a dos narrativas diferentes sobre el fenómeno de la verticalización: una, articulada en torno a las encuestas de edificación, y, la otra, en torno a notas de prensa sobre sucesos en edificios de altura. Ambas son útiles para verificar cómo este fenómeno, agenciado en entidades arquitectónicas, pasa a constituirse en un régimen urbano, es decir, como una configuración normativa capaz de producir modos de orden para la interacción en las ciudades.

Palabras clave verticalización, edificios residenciales de altura, modos de orden, sociología urbana, sociología de la arquitectura

Artículo de investigación

Cómo citar este artículo: Vergara Vidal, J. E. (2020). La verticalización como régimen urbano. El caso de las ciudades chilenas. Cuadernos de Vivienda y Urbanismo, 13. https://doi.org/10.11144/Javeriana.cvu13.vruc 


\section{The Verticalization as an Urban Regime.}

The Case of the Chilean Cities

Abstract The proliferation of high-rise residential buildings in the central and pericentral fabric of the Chilean cities is a recent phenomenon and its intensity leads to ask about the effects it has on the urban interaction. This paper explores the records and accounts giving rise to two different narratives of the verticalization phenomenon: a first one articulated around the surveys of building; and a second one based on press releases telling events in high-rise buildings. Both of them result useful to verify how this phenomenon -occurring under the agency of architectural institutions- becomes an urban regime. That is to say, how it translates into regulations to allow producing modes of order for the interaction in the cities.

Keywords verticalization, high-rise residential building, modes of order, urban sociology, sociology of architecture

\section{A verticalização como regime urbano. 0 caso das cidades chilenas}

Resumo A proliferação das edificações em altura para uso residencial nas áreas centrais e pericentrais das cidades chilenas é um fenômeno recente, cuja intensidade incentiva a questão dos seus efeitos na interação urbana. O presente texto explora os registros e relatos que originam duas narrativas diferentes sobre o fenômeno da verticalização: uma articulada em torno de inquéritos de edificação e a outra em torno das notas de imprensa sobre eventos em prédios de altura. Ambas são úteis para verificar como esse fenômeno, agenciado em entidades arquitetônicas, se torna um regime urbano, isto é, como uma configuração normativa capaz de produzir modos de ordem para a interaçáo nas cidades.

Palavras chave verticalização, prédios residenciais em altura, modos de ordem, sociologia urbana, sociologia da arquitetura 


\section{Introducción}

La verticalización describe un fenómeno donde la tipología arquitectónica predominante en un área urbana, tanto constructiva como visualmente, es la que corresponde a los edificios de altura (Grubbauer, 2010; Graham, 2016; Vergara Vidal, 2017). Pero lo que fue visto como una propuesta conceptual y, por tanto, una forma de planificación urbana en la Ciudad Vertical de Hilberseimer (1999) y en el Plan Voisin de Le Corbusier (1985) es hoy un proceso controversial, justamente en razón de su efecto en el ordenamiento de la ciudad, de sus densidades y de sus prácticas.

En el caso particular de las ciudades chilenas, y en especial de sus ciudades metropolitanas (Santiago, Valparaíso y Concepción) (Instituto Nacional de Estadísticas [INE], 2007a), la verticalización es un fenómeno que ha implicado tanto la densificación de sus paños centrales y pericentrales por medio de edificios residenciales de altura, como una masiva estandarización de sus modos de residencialidad por medio de la consolidación de los departamentos como una tipología de vivienda.

Esto también ha traído consigo una creciente homogeneización de las decisiones materiales y espaciales dentro de las soluciones arquitectónicas, que se expanden al conjunto de la ciudad y se replican en realidades urbanas similares. Y es, precisamente, al estandarizar dichas decisiones donde la verticalización muestra su aspecto más interesante: su configuración como un régimen de prácticas sociomateriales masivo y específicamente urbano.

Por régimen cabe entender una configuración normativa, un modo de orden y de gobierno legítimos que sostienen, aunque no exclusivamente, a la acción en cada situación social (Boltanski y Thévenot, 2006). En este caso, en la situación sociomaterial denominada ciudad metropolitana. Por cierto, la constitución de este régimen en particular no tiene su punto de partida en el actual proceso de verticalización, ni en las operaciones de estandarización que le son propias, sino en ejercicios anteriores de los cuales el actual proceso es su consolidación.

Las versiones anteriores de este fenómeno pueden ser reconocidas en los primeros edificios residenciales de altura o colectivos, construidos a partir de la década de 1920, que seguían los estándares de la vivienda moderna, y en los edificios construidos entre 1950 y 1970, que siguieron la estela de la racionalización de los Centros Integrales de Atención al Migrante (CIAM), de su idea de conjunto y de sus tipologías espaciales.

Si bien los edificios residenciales de altura fueron producidos de manera acotada y bajo propuestas muy heterogéneas en su forma y programa; los edificios colectivos, amparados en las decisiones urbanas de un Estado responsable de la vivienda, resultan un conjunto más homogéneo, pues fueron parte de un proceso que estandarizó las decisiones materiales y espaciales bajo el argumento de la racionalización, lo que los constituyó en tipologías y los expandió a todas las ciudades del país, como ocurre con los modelos 1010 y 1020 de la Corporación de la Vivienda.

En comparación con lo anterior, el proceso actual es más intenso, ya que construye anualmente más edificaciones y departamentos y, a su vez, es más estandarizado, pues ya no está guiado por una 
racionalización estatal, sino por criterios comerciales. Esto se traduce en edificaciones más altas, pericentrales, con mayor densidad y con departamentos más pequeños. La conjunción de estos tres procesos en las ciudades metropolitanas chilenas da como resultado un conjunto masivo que enmarca y da gobierno a la experiencia residencial en su interior, aunque este no está limitado a ellas.

Configurado como un modo de orden que emanaba de las decisiones y prácticas del diseño de una comunidad experta específica (la arquitectura) y que fue escalado al plano urbano por otras comunidades, como las que gobiernan el uso y la propiedad del suelo en las ciudades, el régimen sociotécnico que despliegan los edificios residenciales de altura no solo se constituye como un conjunto de objetos normativos que actúan enlazados o con coherencia, sino que también es capaz de aportar identidad a las poblaciones de actores que caben bajo su gobierno. El resultado es un orden tanto coactivo como cooperativo que afecta a las prácticas de residencialidad en las ciudades.

El presente trabajo aborda los registros y relatos desde los que se configura parte del sentido semiótico de dicho régimen sociotécnico. Su hipótesis es que la constitución de los fenómenos en regímenes emerge de su capacidad de expresar un orden en el mundo de la interacción y en las diferentes escalas en donde esta se desarrolla. Tal capacidad es heterogénea y agregativa, no promueve una sola explicación o una sola lectura del fenómeno y, a su vez, proviene de las distintas comunidades de prácticas, cuyas visiones en conjunto conforman un sentido común, distinto de un sentido único, que es movilizado a través de narrativas sostenidas por estos registros y relatos.

El texto explora los registros y relatos que dan pie a dos narrativas diferentes sobre el fenómeno de la verticalización: una, articulada en torno a las encuestas de edificación, y la otra, en torno a notas de prensa sobre sucesos en edificios de altura. Estas dan cuenta de cómo este fenómeno pasa a constituirse en un régimen urbano. En el primer caso, las encuestas de edificación nos lo cuentan como un fenómeno directamente poblacional. Ellas no registran el aumento de edificios de altura, por ejemplo, pero sí registran el cambio en las decisiones materiales que conlleva construir edificios de altura: el consumo de hormigón se dispara, como también lo hace la altura de las edificaciones y esto ocurre en las ciudades metropolitanas, las de mayor población, a las que les caben mejor estas soluciones arquitectónicas.

La prensa, por su lado, articula un conjunto de datos completamente diferente. Sus registros están pensados para ser leídos por comunidades de prácticas heterogéneas y no especializadas, no como ocurre con las encuestas de edificación, $y$, en ese marco, un término técnico como el de verticalización no es parte del relato. A cambio, los edificios son los protagonistas de una historia en la que incrementan su número con el tiempo y pasan de ser meros escenarios de los sucesos, a ser discutidos y comentados según sus características técnicas y habitacionales o según las controversias en las que se ven involucrados.

Ambas narrativas no son explícitas, sino, más bien, emergentes. Pero, aun desde esa posición secundaria, estas narrativas dan razón de la verticalización como un modo de orden de lo material y como un modo de orden del espacio arquitectural en las ciudades. Sus diferencias no tensionan la percepción del fenómeno, sino que expresan el diámetro que es capaz de cubrir su régimen. Las narrativas son parte del enlazamiento de lo vertical al orden de lo común, que a la larga, es también lo predominante.

En lo que sigue, se expondrán los supuesto teóricos y la metodología utilizada para capturar estas dos narrativas desde sus propios repertorios, así como los resultados de dicho ejercicio, lo cual 
conlleva identificar el orden de los sentidos sociomateriales que cada narrativa propone. Posteriormente, estos resultados serán discutidos en función de la caracterización teórica del régimen urbano que despliegan los edificios residenciales de altura en las ciudades, para, finalmente, concluir con las implicancias de la verticalización en el gobierno sociomaterial de estas ciudades.

\section{Supuestos teóricos y metodológicos}

En el presente trabajo, se entiende como una narrativa a un conjunto de enunciados que organizan un orden de sentido especifico respecto al mundo de la interacción (Law y Mol, 2009; Ingold, 2010). Identificar aquellas narrativas que se vinculaban y/o emergen del fenómeno de la verticalización de las edificaciones fue útil para verificar las formas como este se constituía en un orden de sentidos. Gracias a esto, se lograron identificar dos de estas formas: una, que constituía a la verticalización como un orden de sentidos materiales, y, otra, que la organizaba como un orden espacial. Como se señaló, ambas narrativas son emergentes, vale decir, representan modos de orden no predominantes ni evidentes dentro del escenario interaccional. Sumado a esto, ambas narrativas se articulan en torno a registros, relatos y comunidades de prácticas diferentes.

Las estadísticas nacionales constituyen una narrativa especializada que versa sobre las poblaciones de entidades que son de interés para la gobernanza estatal, local o nacional (Thévenot, 2016). Esta narrativa considera a las entidades humanas en tanto poblaciones, pero no se acota a ellas e incluye también a otros conjuntos de entidades no humanas, como la vivienda, el empleo, las especies animales o las materialidades (Goldthorpe, 2017). Se trata, a su vez, de una narrativa que apela y convoca a comunidades de prácticas expertas, para quienes sus enunciados tienen especial significado en la medida en que le dan forma a un orden práctico del mundo que orienta su acción (Star y Griesemer, 1989).

La producción de esta narrativa radica, en primer grado, en los dispositivos que capturan, inscriben y desplazan la información (Bowker y Star, 1999). Estos instrumentos o formularios desplazan hacia los actores ejercicios métricos que conllevan afirmaciones sobre la realidad material que, para las comunidades de prácticas en torno a ellos, son esenciales y que los definen (Ramos, 2018). En el caso elegido, el fenómeno de la verticalización estaba relatado desde dos formularios consecutivos, pero diferentes. El primero, la Encuesta de Edificación, fue elaborado por el Instituto Nacional de Estadísticas (INE) y fue aplicado por los municipios a quienes construían edificios entre 1990 y 2001; el segundo, el Formulario Único de Estadísticas de Edificación (FUEE), fue elaborado por el INE, el Ministerio de Vivienda (MINVU) y la Cámara Chilena de la Construcción - que agrupa a las empresas constructoras, inmobiliarias y de materiales-, y es aplicado también por los municipios hasta el día de hoy como requisito de recepción de una obra (INE, 2006, 2007b). Si bien entre un instrumento y otro hay una continuidad y, por tanto, similitudes, sus diferencias en los elementos contabilizados y medidos dentro de un edificio, y en la composición de quienes lo elaboraron, expresan cambios en la relación entre la sociedad y lo material.

La narrativa sobre la verticalización, que es posible de organizar a partir de los datos recabados por estos instrumentos, no viene dada por el INE, quien opta por organizarlos como un relato sobre la industria de la construcción al considerar las superficies de edificación autorizadas, sino que está basada en los metros cuadrados de materialidades utilizadas en los muros de las edificaciones de una obra nueva, las cuales están segmentadas por pisos de altura en todas las comunas del país 
construidas entre 1990 y 2016. Esto constituye, por decirlo así, una narrativa emergente, de la cual los datos dan cuenta, pero que no comunican por sí mismos o que sus productores no organizan a pesar de producirla.

Por su parte, la narrativa que se organiza en torno a las piezas de prensa constituye, por sí misma, una estrategia diferente y no sistematiza del fenómeno, pero le da forma en la medida en que los reitera en cada nota en la que alude a él. Su narración se organiza a través de los relatos sobre sucesos protagonizados por o en edificios residenciales de altura, que hace públicos diariamente, pero que son difíciles de reconstruir como un conjunto, ya que no siempre los emisores de estas notas las compilan para una observación posterior.

Para dar cuenta de este segundo tipo de narrativa se consideraron piezas de prensa que hicieran mención a edificios de altura, publicadas en los dos principales medios de prensa escrita del país - los diarios El MercuriolEmol y La Tercera-, entre 2009 y 2017. Para identificarlas y recopilarlas, se utilizó el parámetro "Edifico(s)" en los buscadores internos de los sitios web de estos medios y se recopilaron solo aquellas noticias que hacían mención a casos nacionales. Esto limitó la búsqueda a la disponibilidad de archivos que, en el caso de La Tercera, solo llegan hasta 2009, e identificó 482 piezas publicadas en ambos medios entre enero de 2009 y junio de 2017.

Los registros en que se inscriben las piezas de prensa son epistemológicamente múltiples, sus formas de observar y de narrar los fenómenos dependen de variables como el estilo de él o la periodista que los escribe, del evento principal por el cual se menciona a los edificios de altura, de los intereses editoriales del medio para el cual escribe, entre otros. En comparación con esto, las estadísticas estatales son más acotadas en estos aspectos, dado que atienden a normas estables referidas a su sentido técnico, aunque también pueden ser incididas por elementos contingentes.

Cabe considerar que ambos tipos de datos constituyen información que se desplaza a través de registros que son diferentes porque son funcionales para la coordinación de comunidades de prácticas distintas. En tal sentido, cada método de registro es parte de una técnica situada en un método de conocimiento y traducción propio de tales comunidades (Latour, 1987, 2001). Al llevar estos registros un método similar, como lo son las bases de datos cuyo análisis da pie a este artículo, las diferencias entre ellos se aminoran, pero no es conveniente perder de vista lo antes dicho. Solo se trata de una nueva operación de traducción.

El método utilizado para capturar ambos tipos de datos fue similar. Se identificaron sus registros y estos se sistematizaron en bases de datos que permitiesen su análisis mediante elementos estadísticos descriptivos. Sus resultados se comentan a continuación: en primer lugar se encuentra el modo como las estadísticas constituyen un orden y, luego, como lo hacen las piezas de prensa.

\section{Una narrativa estadística de la verticalización}

Como se señaló anteriormente, el método de registro implicado en las estadísticas estatales es bastante diferente al utilizado por las piezas de prensa, porque proviene de una lectura estandarizada de su entorno y de sus poblaciones (Bowker y Star, 1999). Tanto la Encuesta de Edificación, como el Formulario Único de Estadísticas de Edificación corresponden a instrumentos masivamente desplegados en busca de entidades específicas y de los datos de su composición poblacional. En pos de ello, estos documentos son aplicados en todos los municipios del país al momento de otorgar permisos y recibir obras edificadas. 
Estos instrumentos están diseñados bajo los intereses informacionales de los actores que en conjunto los diseñaron —como la Cámara Chilena de la Construcción o el Ministerio de Vivienda, que suma sus logos al del INE en cada formulario del FUEE_- y, al mismo tiempo, responden a intereses públicos, tanto en su forma de ser comunicados como en la utilidad de la información recopilada, que debiera ser un insumo de políticas sectoriales. Ello se expresa en el tipo de información que capturan y que, si bien son específicos a las edificaciones, no registran información sobre su forma, sus colores o su número de ascensores, y no procesan el número específico de pisos, sino solo si alguna edificación sobrepasa los nueve pisos de altura (INE, 2006, 2007b).

Se entiende que lo anterior produce un relato del cual la forma, el color, el transporte vertical o el número de pisos de altura no son parte. Esa es una cuestión de orden, todos los aspectos no considerados están fuera de él y, por tanto, quedan fuera del régimen urbano que con él se configura. El efecto de esto es fácil de observar en la heterogeneidad de la forma, color y altura de las edificaciones de las ciudades metropolitanas chilenas, pero esto mismo es imposible de reconstruir a partir de los datos, ellos solo refieren a la narrativa que están diseñados para contar.

Por esto, los datos estadísticos no cuentan el fenómeno en cuestión, sino que ordenan lo que se observa de él una vez que este se ha desplazado fuera del alcance de la vista, son una forma de ordenar lo que se dice de él. Por ejemplo, en el caso de las edificaciones construidas en las ciudades chilenas entre 1990 y 2016 (figura 1), lo que estas nos narran es que cada año el proceso edificatorio consume mayores volúmenes de materiales, la mayor cantidad de ellos (71\%) van dirigidos a la construcción de edificaciones de baja altura, el $17 \%$ es utilizado en edificaciones de 9 pisos y más, mientras que el $12 \%$ restante se ocupa en las alturas intermedias de entre 4 y 8 pisos.

El problema de esas afirmaciones es que no tienen forma, como tampoco la tiene el gráfico que las representa (figura 1). Lo que se tiene es sinuosidad, una diferencia sin singularidad capaz de ser narrada por este sistema de orden en particular.

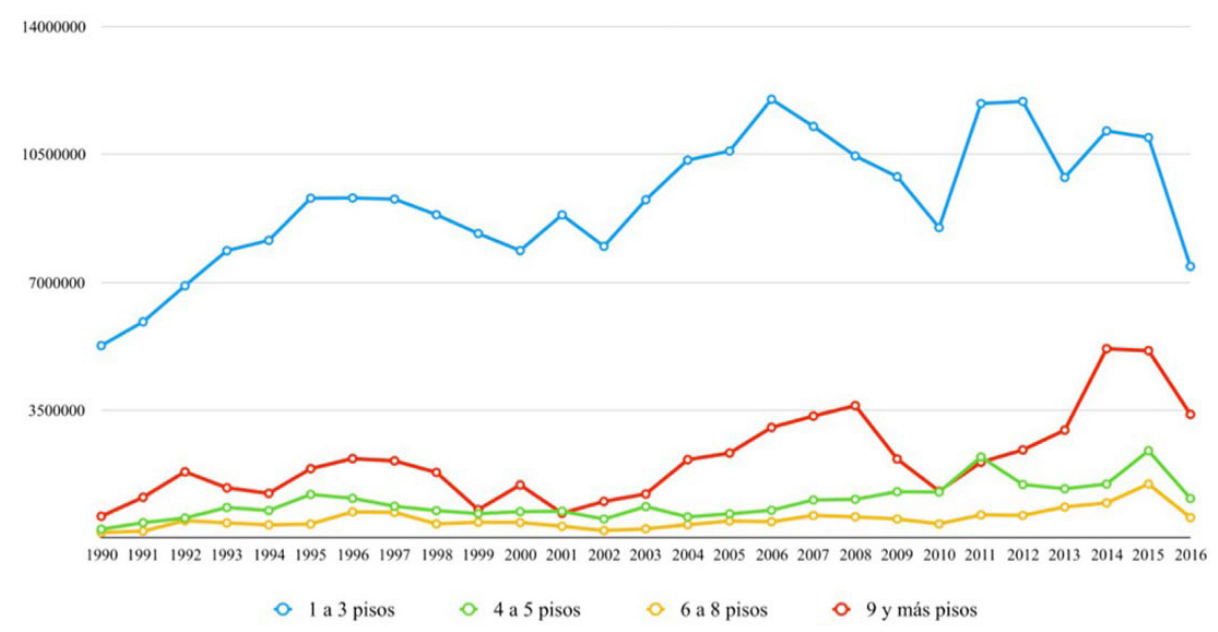

Figura 1. Materialidades utilizadas en los muros de edificaciones de obra nueva en Chile según los pisos de altura y el año $\left(m^{2}\right)(1990-2016)$

Fuente: elaboración propia a partir de los datos de la Encuesta de Edificación (1990-2001) y del Formulario Único de Estadísticas de Edificación (2002-2016) 
Es dentro de este panorama que se encuentran los primeros indicios narrativos de la verticalización, como una diferencia entre las edificaciones que se dibuja en la curva ascendente de los volúmenes de materiales utilizados en aquellas construcciones sobre los 9 pisos y en su separación de las líneas que representan a las edificaciones de mediana altura. Es más, también puede notarse cierta relación entre las evoluciones similares entre la línea que grafica los materiales utilizados en las edificaciones de 1 a 3 pisos, y la que dibuja los de las de 9 y más pisos.

La idea de un orden polar sustentado en las edificaciones es sugerente, pues da cuenta del desmantelamiento de un régimen anterior basado en la densidad orquestada por el Plan Regulador Metropolitano de 1960 y que promovía las edificaciones de altura intermedia. Lo que los datos narran no es una predicción, es la constatación de que las soluciones residenciales o están en la baja altura, y en la agregación horizontal de las unidades, o están en la altura, y en la agregación vertical que requiere, además, de un gobierno colectivo en la escala arquitectural.
Si lo anterior sugiere a la verticalización como un régimen sociotécnico emergente, las figuras 5 y 6 relatan en su ubicación el contenido marcadamente metropolitano del fenómeno. Si se consideran exclusivamente las edificaciones sobre los 6 pisos, el 83,5\% de las materialidades ocupadas en ellas durante el periodo observado tiene como destino las tres ciudades metropolitanas del país y, dentro de estas, la distribución es igualmente desigual (figura 2). El 69,9\% del total de las materialidades ha tenido como destino comunas del Santiago Metropolitano, mientras que solo el 9,7\% ha sido ocupado en la verticalización del Valparaíso Metropolitano y el 3,9\% en la verticalización de las comunas del Concepción Metropolitano.

Esta última cifra es muy parecida a la que presenta Antofagasta (figura 3), que, según los datos, utilizó en sus edificios de altura el 3,7\% de los materiales destinados a ellos a nivel nacional en el periodo observado. Junto a ella, otras 5 ciudades narran una historia de la verticalización que va a la par de sus propios procesos de metropolitanización. Estas 6 ciudades no siguen tendencias

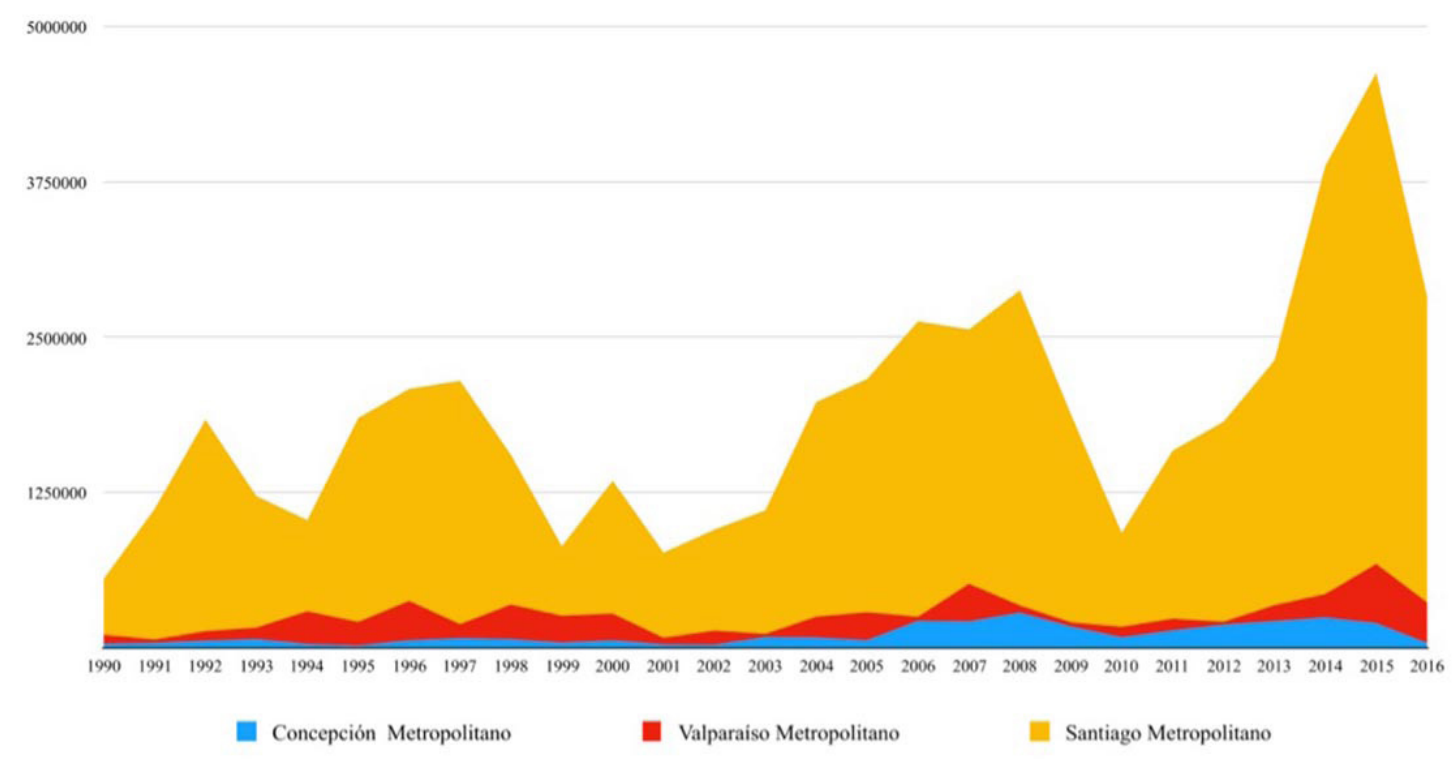

Figura 2. Materialidades utilizadas en los muros de las edificaciones de 6 pisos y más de obra nueva en ciudades metropolitanas de Chile $\left(\mathrm{m}^{2}\right)(1990-2016)$

Fuente: elaboración propia a partir de los datos de la Encuesta de Edificación (1990-2001) y del Formulario Único de Estadísticas de Edificación (2002-2016) 
estables ni similares, aunque todas tienden a presentar mayores consumos tras el año 2010.

Antofagasta es la que presenta mayores consumos de materiales, sobre todo durante el periodo 2013 y 2014, seguida por la ciudad conurbada de Iquique-Alto Hospicio, cuya curva expresa el 2,8\% de las materialidades nacionales dentro del periodo observado, mostrando un aumento persistente de consumo, y por la conurbación La Serena-Coquimbo, que explica el 1,8\% de los consumos ya seńalados. En todos estos casos, hay aumentos en el consumo de material para las edificaciones en altura durante el año de 1991, en torno al año de 1997, una década después en el 2007 y luego en torno al 2014, lo cual puede obedecer a una tendencia también presente en las ciudades metropolitanas (figura 3).

La conurbación de las comunas de Temuco-Padre Las Casas (figura 3), la única de estas ciudades que no es costera, explica el 1,3\% de los consumos materiales en edificaciones de altura del país y parece seguir un ritmo de consumo a contrapelo de los antes vistos; mientras que Algarrobo es la única de estas ciudades que no corresponde a una ciudad intermedia, sino a una ciudad costera de uso vacacional. Con una población que supera levemente los 10.000 habitantes, Algarrobo ocupa el sexto lugar de esta selección, desplazando a ciudades como Copiapó-Tierra Amarilla, que cuenta con 170.000 habitantes, y Rancagua, poblada por 250.000 personas. La razón es numérica, aunque cada una de estas tres ciudades representa el $0,6 \%$ de los consumos materiales destinados a las edificaciones de altura en el país durante el periodo observado, el consumo de Algarrobo supera en 10.000 metros cuadrados de materialidades el consumo de Copiapó-Tierra Amarilla y en 20.000 metros cuadrados el de Rancagua, lo que puede deberse al uso turístico de las edificaciones de altura de Algarrobo.

Este dato indica que la verticalización no solo es un orden de procesos de densificación urbana, sino que también son modos de residencialidad que merecen ser desplazados desde las altas densidades de las ciudades metropolitana hacia las costas, donde los segmentos de mayores ingresos vacacionan y/o mantienen segundas residencias.

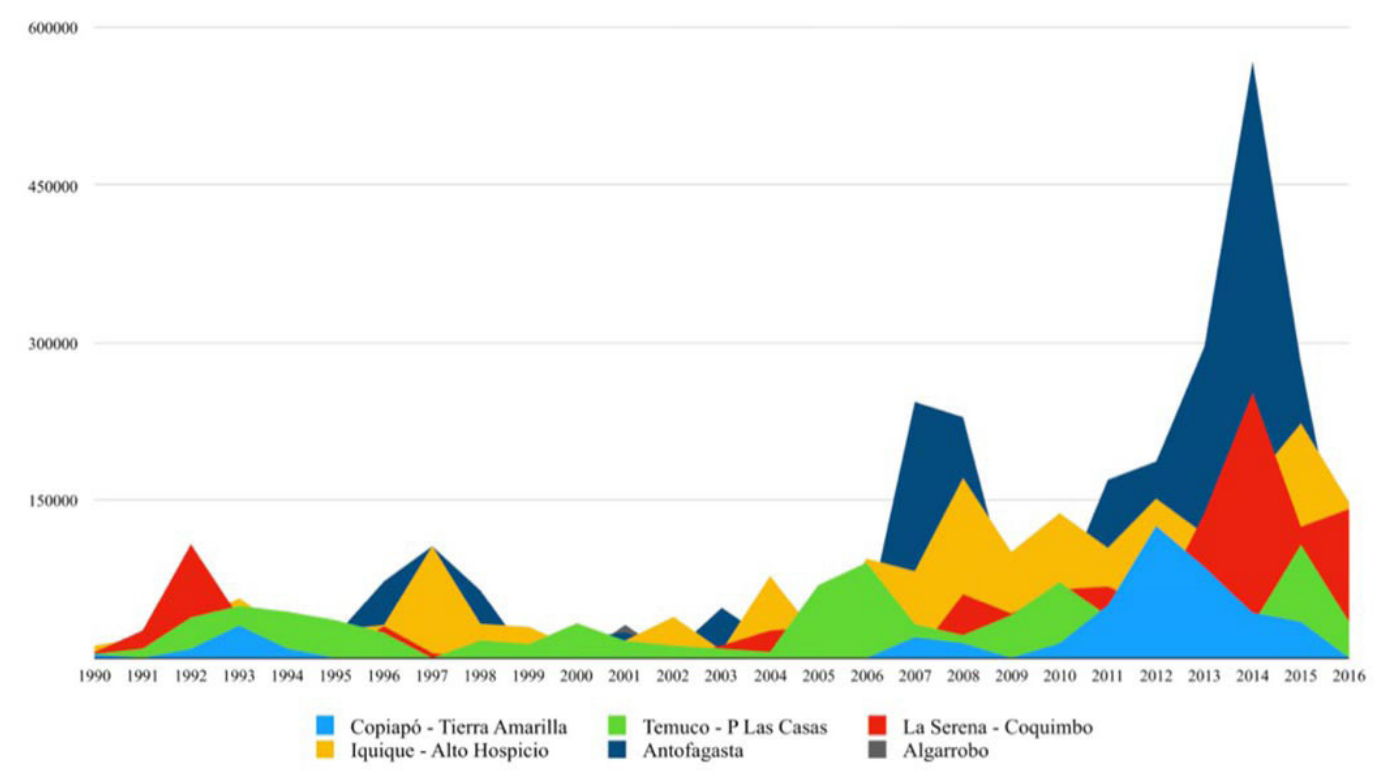

Figura 3. Materialidades utilizadas en los muros de edificaciones de 6 pisos y más de obra nueva en las ciudades metropolitanas de Chile $\left(\mathrm{m}^{2}\right)$ (1990-2016)

Fuente: elaboración propia a partir de los datos de la Encuesta de Edificación (1990-2001) y del Formulario Único de Estadísticas de Edificación (2002-2016) 
Los edificios se muestran como una solución material y espacial más atractiva que las casas, incluso cuando se trata de una casa de playa, aunque su ubicación está altamente concentrada, dado que estas 9 ciudades concentran el 93,8\% de las materialidades utilizadas en edificaciones de altura.

En el resto de las ciudades del país, el fenómeno se desarrolla de forma menos regular, no superando en ninguna ciudad el $1 \%$ de participación del total y en su conjunto solo alcanza el 6,2\%. De las 291 ciudades observadas, 88 presentaron consumos materiales destinados a edificaciones mayores de 6 pisos entre 1990 y 2016, pero solo 40 presentaron consumos superiores a los 20.000 metros cuadrados de materiales en muros en los últimos 26 años.

Estos datos narran el fenómeno de la verticalización de la edificación como un orden urbano, muy metropolitano, por cierto, pero capaz de desplazarse con sus ocupantes metropolitanos si estos lo requieren, como es el caso de Algarrobo o Iquique-Alto Hospicio, es decir, como un régimen de orden que se desplaza con los habitantes a quienes les es funcional. Así, este fenómeno se muestra, no solo como una forma de construir de las grandes ciudades, sino como un modo de habitar que se desplaza a partir de ellas.

\section{Una narrativa periodística de la verticalización}

Ni los instrumentos ni las bases de los cuales provienen los datos comentados anteriormente estaban diseñados para ser sensibles al fenómeno de la verticalización; por lo mismo, cabe considerar la intensidad que este fenómeno ha tenido para hacerse notar dentro de un marco narrativo que no lo tenía presupuestado.

La prensa representa un marco narrativo sensible a la ocurrencia de lo nuevo, de lo extraño o de lo inusual, pero sin la capacidad de dar una imagen comprensiva del fenómeno. Su modo de ordenarlo es desplazarlo hacia lo público, mediante la iteración y/o la espectacularidad de los sucesos narrados (Thompson, 2001). En tal sentido, su métrica, basada en la intensidad del suceso y en la experiencia que de ello es posible comunicar y desplazar, configura un modo de orden mucho más contingente, una narrativa en constante reconfiguración de su orden semántico, pero que también es capaz de trazar continuidades y causalidades en procesos de largo plazo (Mahoney, 2012).

Un ejemplo de este orden narrativo, basado en la continuidad o persistencia de cierto tipo de

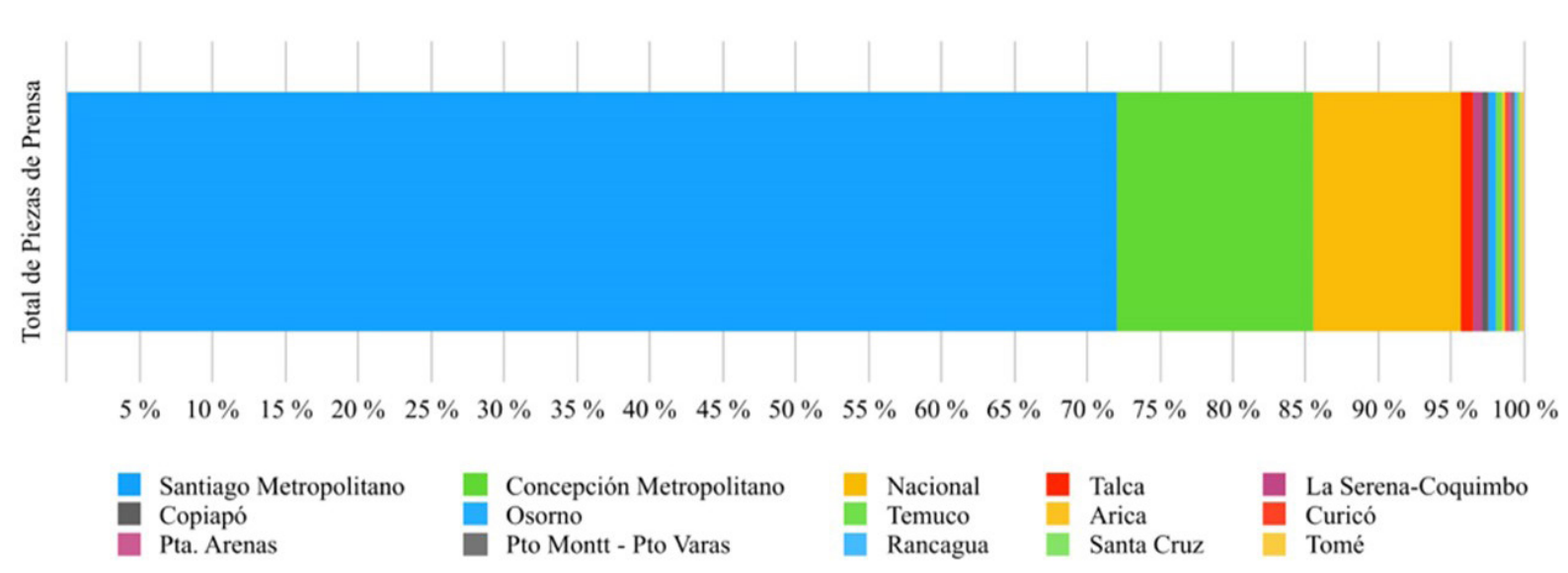

Figura 4. Porcentajes de piezas de prensa sobre los edificios de altura según la ciudad a la que hacen mención entre enero de 2009 y junio de 2017

Fuente: elaboración propia a partir de los datos de piezas de prensa, 2009-2017 
sucesos, es lo que se pudo obtener luego de identificar y sistematizar 482 piezas de prensa que mencionan y tratan sobre edificios de altura, publicadas en 2 medios de prensa - La Tercera y El Mercurio - entre los años 2009 y 2017. En piezas de prensa, se corroboran las tendencias vistas antes en la narrativa estadística, como el carácter metropolitano del fenómeno. Si se considera la ciudad en que sucede el suceso relatado, el 67\% de estas piezas se ubica en comunas del Santiago Metropolitano, el 5,5\% en comunas del Valparaíso Metropolitano y el $12,5 \%$ en comunas del Concepción Metropolitano (figura 1).

Estas tres ciudades metropolitanas dan orden geográfico al 85,5\% de las piezas de prensa observadas. Como efecto de esta concentración, y coincidiendo con lo expresado por los datos de distribución de las materialidades, solo el 4,1\% de las piezas de prensa relatan hechos sucedidos en otras ciudades del país, principalmente en las ciudades conformadas por las comunas de Antofagasta, Iquique-Alto Hospicio, La Serena-Coquimbo, Temuco-Padre Las Casas, Copiapó-Tierra Amarilla, Osorno y Talca.
Cabe consignar también que un 9,4\% de las piezas de prensa versan sobre eventos de escala nacional, el cual es un orden que se da solo como agregación en el caso de la narrativa estadística. En este caso, las piezas de prensa, más que relatar eventos, hacen mención a la legislación, normas técnicas, regulaciones o aspectos que se aplican al conjunto de los edificios de altura del país (figura 4).

En lo que se refiere al modo del orden temporal de estas piezas en el periodo considerado (20092017), es posible observar que los años en que se produjeron más piezas de prensa relacionadas con edificaciones de altura son el ańo 2010, que presenta un 19,1\% del total; seguido por el año 2017 , con un $16 \%$, aun cuando solo se consideraron 6 meses de este año; y el año 2013, donde se emitió el 14,6\% de las piezas de prensa recopiladas (figura 5).

Estos tres aumentos en la producción de piezas de prensa relacionadas con los edificios de altura tienen explicaciones diferentes. El aumento del año 2010 se explica por el terremoto (figura 6) y, por lo mismo, aumentan las menciones de las ciudades que protagonizaron este evento, como

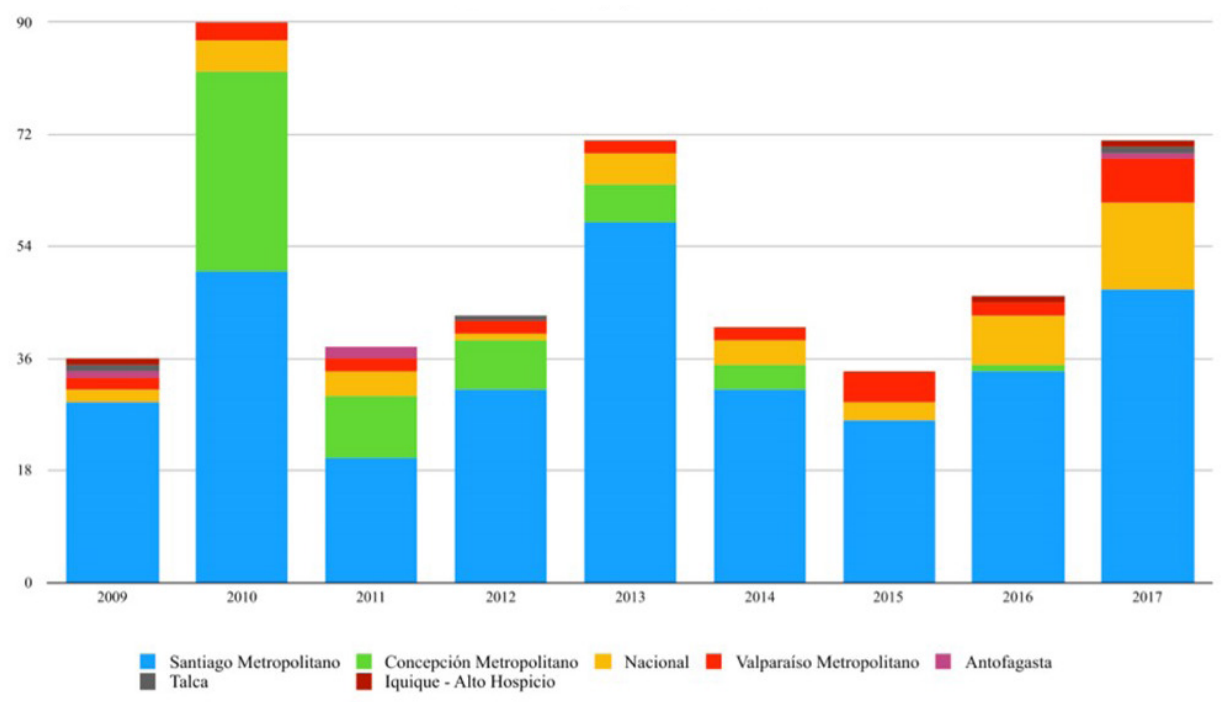


son las comunas del Concepción Metropolitana y la ciudad de Talca, puesto que en ellas no solo se produjeron daños en edificios de altura, sino que también se relataron un mayor número de reparaciones, demoliciones y procesos judiciales asociados a este evento. Este tipo de menciones decrece hacia el año 2014 (figura 5).

Por su parte, el aumento en el 2013 obedece a un alza de las inversiones inmobiliarias, la construcción de edificios de altura y de los accidentes en estos. Mientras que el aumento en las piezas de prensa del año 2017 responde a la controversia de los guetos verticales, que se verá más adelante, la cual aumentó las menciones de las comunas del Santiago Metropolitano y las notas referidas a la escala nacional, a la que se asocia la planificación urbana. Cabe señalar también que esta controversia motivó una escalada de cancelaciones de permisos de construcción de edificaciones de altura en ciertas comunas de Valparaíso y Santiago, lo que sumó menciones a estas ciudades (figura 5).

La diversa naturaleza de los eventos que relatan las piezas de prensa permite configurar un modo de orden basado en su agrupación temática, lo que hace posible 7 categorías: "Accidentes", "Ciudad", "Delitos", "Mercado inmobiliario", "Tecnología", "Destino del edificio" y "Terremoto", esta última está asociada al sismo de febrero del 2010. Además de estas 7 categorías, es posible distinguir otras 33 subcategorías que se detallan en la tabla 1.

La categoría de "Accidentes" es la que agrupa la mayor cantidad de piezas de prensa $(38,1 \%)$. Se trata de piezas que hacen mención a accidentes relacionados y/o ocurridos en edificios de altura, en especial, caídas voluntarias o involuntarias desde ellos $(10,6 \%)$, incendios $(14,9 \%)$ y accidentes en ascensores $(4,1 \%)$. Las piezas relacionadas con eventos vinculados al "Terremoto" del 2010 articulan una segunda categoría y agrupan al $18,4 \%$ del total de piezas observadas, dentro de las cuales tienen especial participación aquellas que hacen mención de los procesos de diagnóstico, reconstrucción y reparación de los edificios afectados $(8,6 \%)$ y los procesos judiciales que tuvieron ocurrencia con posterioridad al terremoto $(7 \%)$.

La tercera categoría en términos de participación porcentual es la que agrupa noticias sobre el mercado inmobiliario $(14,5 \%)$ y que versa sobre la venta y compra de edificaciones y departamentos y las localizaciones de mayor rentabilidad. Por su parte, la categoría que agrupa temáticas relacionadas con "Tecnología" en los edificios de altura captura el $11,9 \%$ de las piezas, siendo las más relevantes las noticias que tienen que ver con el habitar, como normativas y controversias con la naturaleza (inundaciones en bordes de ríos y del mar) $(5,7 \%)$ y avances técnicos en la construcción $(2 \%)$ y provisión de energía domiciliaria (2\%).

Las piezas de prensa relacionadas con noticias y controversias sobre el "Destino" público, privado o patrimonial de los edificios de altura conforma una quinta categoría que agrupa al 6,9\% de las piezas observadas, dentro de las cuales las noticias sobre edificios de destino público explican el 3,7\% y las controversias y noticias sobre los edificios de destino patrimonial explican el $1,8 \%$, mientras que las noticias sobre los edificios de destino privado capturan el 1,4\% del total.

Las piezas de prensa que están agrupadas en la categoría "Ciudad" (6,1\%) corresponden a textos donde el tema central gira en torno al problema de la densidad de las edificaciones de altura y su efecto en el entorno urbano $(4,1 \%)$ o la vialidad $(0,2 \%)$, los permisos de edificación otorgados o quitados (1\%), o la propia altura de las edificaciones $(0,8 \%)$. Por último, la categoría de "Delitos" $(3,6 \%)$ agrupa las piezas relacionados con 
el relato de crímenes de sangre $(1,4 \%)$, comercio o acoso sexual $(0,2 \%)$, robos $(0,4 \%)$, bombas explosivas en edificios públicos $(0,6 \%)$ o tomas de edificios públicos $(0,6 \%)$.

Tabla 1. Distribución de las temáticas centrales en las piezas de prensa observadas sobre edificios de altura de enero de 2009 a junio de 2017

\begin{tabular}{|c|c|c|}
\hline Categoría & Temáticas & $\%$ \\
\hline \multirow{9}{*}{$\begin{array}{l}\text { Accidentes } \\
38,1 \%\end{array}$} & Ascensor & 4,1 \\
\hline & Caída & 10,6 \\
\hline & Choque & 0,2 \\
\hline & Delito & 1 \\
\hline & Derrumbe & 2 \\
\hline & Emergencia química & 2,9 \\
\hline & Explosión & 1 \\
\hline & Gas & 1,4 \\
\hline & Incendio & 14,9 \\
\hline \multirow{4}{*}{$\begin{array}{l}\text { Ciudad } \\
6,1 \%\end{array}$} & Altura & 0,8 \\
\hline & Densidad & 4,1 \\
\hline & Permisos de edificación & 1 \\
\hline & Vialidad & 0,2 \\
\hline \multirow{5}{*}{$\begin{array}{l}\text { Delitos } \\
3,6 \%\end{array}$} & Sexual & 0,2 \\
\hline & Bomba & 1 \\
\hline & Crimen de sangre & 1,4 \\
\hline & Robo & 0,4 \\
\hline & Tomas & 0,6 \\
\hline $\begin{array}{l}\text { Mercado } \\
\text { inmobiliario } \\
14,5 \%\end{array}$ & Mercado inmobiliario & 14,5 \\
\hline \multirow{7}{*}{$\begin{array}{l}\text { Tecnología } \\
11,9 \%\end{array}$} & Ascensor & 0,8 \\
\hline & Bicicletas & 0,8 \\
\hline & Construcción & 2 \\
\hline & Energía & 2 \\
\hline & Habitar & 5,7 \\
\hline & Protesta & 0,4 \\
\hline & Reciclaje & 0,2 \\
\hline \multirow{4}{*}{$\begin{array}{l}\text { Terremoto } \\
18,4 \%\end{array}$} & Procesos judiciales & 7 \\
\hline & Reconstrucción & 8,6 \\
\hline & Robo & 1 \\
\hline & Terremoto & 1,8 \\
\hline \multirow{3}{*}{$\begin{array}{l}\text { Destino } \\
6,9 \%\end{array}$} & Edif. Patrimonial & 1,8 \\
\hline & Edif Público & 3,7 \\
\hline & Edif. Privada & 1,4 \\
\hline
\end{tabular}

Junto al orden categorial de las piezas de prensa hay también un orden temporal que se puede articular en el periodo observado (2009-2017) (figura 6) y que muestra una predominancia estable de los sucesos considerados como "Accidentes" sobre los agrupados en otras categorías. Obviamente, en el año 2010 las piezas de prensa que hablaban sobre el terremoto capturaron casi el $50 \%$ de las menciones de edificios de altura de ese año, pero, en los años siguientes, la participación porcentual de esta categoría desciende, dando pie a que otros temas sean los que dominan las discusiones (figura 6).

Por ejemplo, las noticias relacionadas con el "Mercado inmobiliario" crecen notablemente en los años 2013 y 2017, en los que la discusión giró alrededor de los efectos posibles y reales del aumento del IVA para la compra de departamentos. Las noticias sobre temas de tecnología también tuvieron una subida en el mismo momento en que la discusión inmobiliaria antecedía al boom constructivo del 2013 al 2015. De igual forma, ambos temas se dispararon en el año 2017, así como las discusiones sobre los usos de los edificios y sobre los accidentes en los edificios de altura, los cuales han fluctuado entre el 15\% y $30 \%$ de las notas de prensa (figura 6).

Un punto aparte merecen las piezas de prensa asociadas a las temáticas de la "Ciudad", que han incrementado su numero en el año 2017 más que en todo el periodo observado y que alcanzan el $17 \%$ de las piezas de ese ańo, siendo superadas solo por las piezas de prensa relacionadas con el "Mercado inmobiliario". Esto tiene relación con la discusión sobre la densidad poblacional de los edificios (guetos verticales) y las consecuentes acciones de algunos gobiernos locales para limitar su altura y revocar permisos de edificación (figura 6). 


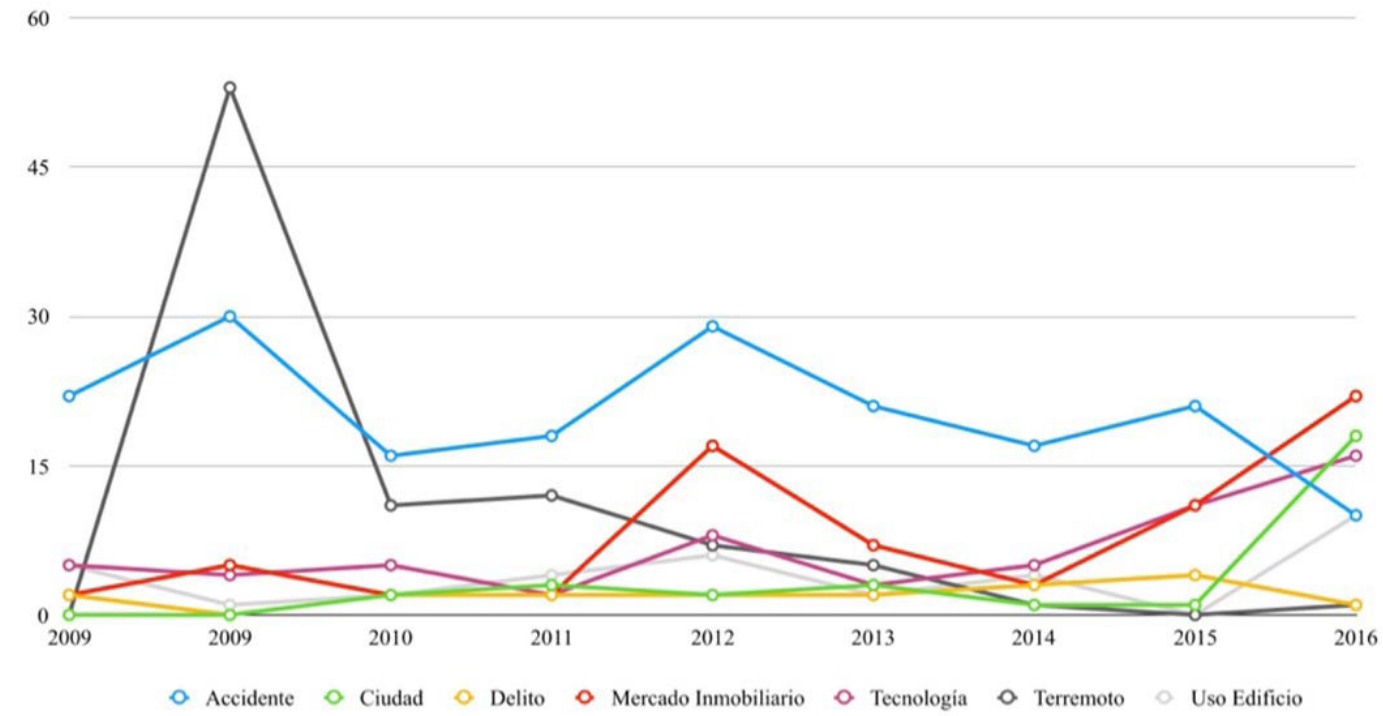

Figura 6. Evolución de las temáticas centrales de piezas de prensa sobre los edificios de altura observadas entre enero de 2009 y junio de 2017

Fuente: elaboración propia a partir de los datos de piezas de prensa, 2009-2017

Los modos de orden posibles en los que se puede articular el conjunto de piezas de prensa observadas, corroboran el sentido metropolitano que se le da al fenómeno de la verticalización y la escasa identificación técnica del mismo y de los edificios de altura en general. En la mayoría de las piezas de prensa, los edificios solo son notados cuando son el escenario de accidentes, delitos, acciones de mercado o catástrofes como los terremotos. Es menor la cantidad de eventos en los que estos son relevados por su acontecer técnico y/o arquitectónico, lo que los configura, en las piezas de prensa, como un orden espacial metropolitano, más que una forma tecnológica en específico. Esto denota los marcos epistemológicos en los que se desarrolla el debate social sobre el fenómeno de la verticalización, y el modo de orden bajo el cual este y sus componentes son gestionados.

\section{La verticalización como un problema de orden epistemológico}

Las formas como la verticalización se configura en un régimen urbano tienen relación con los repertorios que agencian sus modos de ordenar el mundo de la interacción. En los apartados anteriores, se han revisado dos de estos repertorios, que han sido caracterizados como "narrativos" debido a que expresan cierta mecánica semiótica tras sus formas de ordenar el mundo sobre la base de diferencias funcionales. En este sentido, el fenómeno de la verticalización se caracterizará por las diferencias que componen sus repertorios materiales (concreto), edificatorios (pisos en altura), residenciales (departamentos), espaciales (pasillos, halls, terrazas), tecnológicos (ascensores, bombas de agua), entre otros, respecto de los repertorios propios de las edificaciones de baja y mediana altura, utilizados en las ciudades.

El conjunto de estos repertorios se encuentra comprendido en la tipología del edificio residencial de altura, puesto que no es a través de su singularidad, sino de su estandarización, que se multiplican sus efectos de orden. Al proliferar los casos tipológicos, se asiste a la apuesta en escena de un régimen estandarizado, es decir, capaz de coordinar comunidades de prácticas diferentes 
y de replicarse en ciudades con climas y geografías distintas.

Que tal proceso solo pueda ser apreciado como un conjunto a partir de sus repertorios materiales, dado que no hay estadísticas sobre el número de edificaciones de altura en el país, es un indicador que, como los repertorios de la tipología, es útil para configurar una narrativa sobre la misma, vale decir, un orden de sentidos que le es particular. Algo similar ocurre en el asunto de las piezas de prensa, donde es posible notar cómo los casos narrados desplazan vivencias metropolitanas, como un problema con los ascensores, a ciudades que no tienen similares características de tamaño o de población, o cómo aumenta la notoriedad de la tipología cuando esta se muestra como una vulnerabilidad urbana, lo que ocurre en el marco del terremoto de 2010.

Sin embargo, no estamos frente a un orden urbano consolidado. Los repertorios observados constituyen modos de orden emergentes capaces de gobernar prácticas de habitar urbano, pero estos no son la base de una planificación, sino, más bien, son el efecto no solicitado de un proceso de densificación pensado solo desde la oportunidad de obtener ingresos. Esto fue lo que el mercado inmobiliario significó para las rentas municipales. En tal sentido, estamos frente a un régimen económico que promueve, no un régimen arquitectural o sociotécnico del habitar. Este es un modo de rentabilizar los pańos centrales de las ciudades, incrementando verticalmente el stock de vivienda disponible (Martínez y López, 2018; Marín, Ruiz-Tagle, López-Moralez, Orozco y Monsalves, 2019).

La incapacidad del modo de orden económico, que da origen al actual proceso de organización, para plegar a los conjuntos sociotécnicos implicados en la tipología arquitectónica de los edificios de altura, da pie a desórdenes y controversias que pueden ser notadas en las piezas de prensa que los evidencian, tanto como patrones como singularidades casuales.

Por ejemplo, durante las primeras semanas de abril de 2017, tres situaciones controversiales fueron cubiertas por los medios de comunicación escrita y cada una de ellas daba cuenta de un modo de orden a los que eran asociados edificios de altura. La primera apelaba a una controversia entre el orden económico y el orden técnico del edificio, y versaba sobre una torre de 21 pisos ubicada en el centro de una ciudad intermedia (Rancagua) cuyos 2 únicos ascensores llevaban ya 2 meses sin funcionar debido al costo de sus reparaciones: uno había cesado de operar en el 2010 y el otro en febrero del $2017^{1}$. Esto había dejado a sus habitantes prácticamente encerrados en las alturas.

La segunda situación daba cuenta de una controversia sobre el modo de orden basado en el uso, que afectaba a los edificios de altura en una comuna pericentral y de altos ingresos del Santiago Metropolitano. La nota de prensa relataba la acción de la municipalidad por asegurar que los edificios residenciales de la comuna no tuviesen un uso asociado con el comercio sexual, para lo cual habían organizado operativos policiales "edificio por edificio", con el fin de disuadir la demanda y disminuir los delitos alrededor de la oferta sexual ${ }^{2}$.

La tercera situación relatada en las piezas de prensa giraba en torno a densidad poblacional de un conjunto de 6 edificios residenciales de 42 pisos de altura, cada uno con 500 departamentos de 30 metros cuadrados en promedio, servidos por tres ascensores y aceras de un metro de ancho ${ }^{3}$. Esta configuración llevaba a que este estándar de hábitat residencial fuera cuestionado como "subnormal" o "precarizado", considerando a estos lugares como "infraviviendas" y 
denominando a los edificios que las contenían como "guetos verticales"

Estos aspectos no tuvieron un gran protagonismo en un debate que privilegió la discusión sobre los beneficios de la localización que daba la ubicación de las torres ${ }^{5}$, la libertad de elección que imperaba en quienes decidían comprar y vender allí, y la necesidad de la autorregulación de las empresas a la hora de diseñar edificios con estas características ${ }^{7}$. A días de iniciada la controversia, el municipio, cuestionado por su falta de regulación, decidió, al igual que otros municipios como Independencia, Santiago y Recoleta, limitar la altura de los futuros edificios de la comuna, sin pronunciarse sobre otros aspectos del diseño como las aceras, la cantidad de ascensores y el tamaño de los departamentos, porque, con todo, los edificios estaban construidos dentro de lo que la norma legal exigía. El problema se desplazó, entonces, hacia la esfera de la ciudad, su planificación y la producción de un soporte urbano para este tipo de arquitectura.

Las 3 situaciones señaladas ilustran la heterogeneidad de los conjuntos de orden a los que los edificios de altura están adscritos. En este caso, de acuerdo con su operación técnica, a su programa de uso, a la calidad de su integración, al conjunto urbano o a escala arquitectural o urbana, sin que predomine con claridad alguna de ellas.

Esto ayuda a la indeterminación objetual de los edificios. En cada una de las 3 situaciones mencionadas, los edificios de altura funcionan de una manera en la que se desacoplan de lo esperado y quedan desenlazados de la forma de actuar que se les requiere. En una de las situaciones no funcionan como una unidad operativa; en la otra, no funcionan (solo) como una unidad residencial; y, en la última, no funcionan como una unidad de integración, debido a que los actores que viven en ellos o que los administran los llevan hacia determinados modos de orden y los desplazan de otros.

Tanto la diversidad temática de las piezas de prensa como las controversias vistas acerca del funcionamiento de los edificios residenciales de altura muestran que sus adscripciones a órdenes son contingentes. Incluso aquellos modos de orden que son parte de sus repertorios propios, como la residencialidad, pueden ser desarticulados si un orden basado en la propiedad y en la libertad de uso los dispone de otra forma.

Esto los presenta como entidades capaces de desenlazarse con facilidad de los modos de orden requeridos, dando pie a que se considere que incrementan la vulnerabilidad de las ciudades, como en general ocurre con las técnicas que son empleadas por órdenes epistemológicos diferentes (Hommels, 2005). Pero esta vulnerabilidad no es intrínseca a la tipología o a sus casos, sino que tiene que ver con que, aun cuando la verticalización es vista como un fenómeno urbano, no se le entiende como un régimen urbano, haciendo persistente su invisibilidad.

Desconocer el número y el funcionamiento de los edificios de altura en una ciudad es un efecto epistemológico inscrito en el diseño de sus instrumentos y de su procesamiento, que enmarca la compresión de los fenómenos. Otros instrumentos sufren efectos distintos, contabilizan y guardan la información faltante relativa a lo que sucede en otras escalas, pero no es posible notar un enlazamiento efectivo entre unas y otras prácticas de información. Prácticas que tienen que ver con la administración de los flujos y las movilidades dentro de los edificios, la gestión del agua y su presión, el mantenimiento de los pisos y los ascensores, entre otros, van quedando inscritas en registros (libros de administración, bitácoras, carpetas, archivos) que dan cuenta de las vicisitudes de la individualidad técnica de los 
edificios, pero estos registros no escalan con facilidad hacia a lo urbano (Blečić y Cecchini, 2017).

Esa falta de enlazamiento o de coordinación de los datos y de sus prácticas, que colabora con una particular vulnerabilidad de la gobernanza urbana, persiste mediante el supuesto epistemológico de que se está frente a dos fenómenos (ciudad/edificios) y no solo frente a uno. Para John Law (2015), las diferentes epistemologías aplicadas sobre un mismo objeto de observación dan como resultado visiones diferentes que nos informan sobre los modos de existencia de una misma entidad técnica. El carácter "real" de cada una de las visiones epistemológicas es comprobable en la medida en que cada una de ellas motiva prácticas específicas, concretas y reales: tienen efecto en la realidad (Law y Mol, 2009), ya se exprese esto en las prácticas que conlleva la aplicación de una regulación legal, en aquellas que devienen del mantenimiento o en otras.

El problema no radica en la diversidad epistemológica de estas prácticas, sino en la falta de coordinación entre ellas. La ausencia de coordinación entre las prácticas es, en el fondo, una ausencia de acuerdo sobre la realidad observada, sobre las entidades que la habitan y sobre las formas de interactuar con ellas (Latour, 2001; Law y Mol, 2009) y, en el caso visto, ello implica una falta de acuerdo o una controversia sobre la comprensión del fenómeno en cuestión y sobre las formas de su gestión.

Esto no es un producto de la condición epistemológica de los objetos (edificios), sino de la ausencia de coordinaciones contingentes de las prácticas, incluidas aquellas que nos informan sobre ellos (Law, 2015). Como se ha visto, las escalas urbana y arquitectónica se nos presentan como planos no conectados entre sí, en cada uno de las cuales un mismo objeto observado (el edificio de altura) se comporta de forma diferente: en una como un fenómeno (urbano) y en la otra como un objeto (arquitectónico). Si esto obedece a un problema de coordinación entre las prácticas de observación de la realidad, sería necesario que estas logren establecer una conexión entre las escalas que nos muestre un objeto que "existe" en ambas (Law y Mol, 2009).

Una posibilidad de establecer este enlazamiento entre escalas podría ser a través de un tipo de práctica presente en todas las escalas identificadas, una suerte de cuerda que permita continuar la observación o la generación de datos, sin el efecto interruptor del cambio de escala. Las prácticas sincréticas, identificadas por Law y Singleton (2013) como formas de componer una visión coordinada sobre los objetos, sin negar su heterogeneidad y/o su multiplicidad epistemológica, podrían cumplir con tales estas características. En realidad, Law y Singleton sostienen que todas las prácticas son sincréticas y proponen 6 modos en los que el sincretismo funciona en lo concreto, mediante la negación, la domesticación, la separación, el cuidado, el conflicto y el colapso. En cada uno de estos modos, el sincretismo ayuda a enlazar las entidades o los objetos en asociaciones y constituye formas de coordinar su inclusión en lo social, pero, además, es posible verificar la presencia de prácticas sincréticas en todas las escalas, como ocurre, por ejemplo, con las prácticas de cuidado que se expresan de distinta forma (mantenimiento, normativas, entre otras) en todas ellas.

Se hacen necesarios métodos de observación a nivel agencial y seguimientos diacrónicos de practicas de sincretismo para articular una visión multiescalar del fenómeno de la verticalización urbana y de los puntos de coordinación que son posibles de establecer. En pos de ello, resulta interesante aproximarse a los edificios de altura no solo como componentes de un fenómeno de escala estructural, como lo es la "verticalización", sino también como un tipo especial de objetos 
(Latour, 1987; Guggenheim, 2009; Blečić y Cecchini, 2017) y/o un tipo especial de tecnología (Guggenheim, 2009) y/o un tipo especial de orden urbano (Graham, 2016; Vergara Vidal, 2017)

\section{A modo de conclusión: la verticalización y el orden urbano}

Como se señaló anteriormente, las dos narrativas analizadas son modos emergentes que permiten situar en una configuración de orden al fenómeno de la verticalización y, a la vez, de expresarlo como un modo que ordena, bajo un sentido identificable, tanto un conjunto de prácticas materiales como un conjunto de sucesos que ocurren en las ciudades chilenas.

Considerado como una forma de orden material, la verticalización de las edificaciones también es capaz de mostrar un sentido infraestructural, pues no se trata de formas aleatorias, sino de un conjunto tipológico que, dentro de su heterogeneidad, obedece a patrones cuya iteración está asegurada por la estandarización inherente a las tipologías, ya se traten de edificios o de sus unidades internas, como departamentos, lobbies, salas de ascensores, calderas, entre otros.

Este mismo sentido de orden, radicado en la condición infraestructural, está latente en los sucesos narrados en las piezas de prensa en tanto espacialidad, porque, finalmente, el conjunto de temáticas identificadas lo que muestra es la heterogeneidad de una misma forma de espacio, aquella construida verticalmente.

El punto donde se tocan las dos narrativas emergentes observadas es aquel que identifica a la verticalización, más que como un fenómeno que resulta de la suma de sus partes (edificios), como uno que va más allá de eso desde lo arquitectural a lo infraestructural, de manera que el efecto conjunto de una tipología resulta en un modo de orden adscrito a los contextos urbanos en los que se desarrollan, en este caso, contextos metropolitanos.

Lo interesante de este aspecto es la capacidad de desplazamiento que demuestra lo metropolitano, en tanto modo de orden particular, a través de los edificios. El caso de Algarrobo, Antofagasta y de la conurbación de Iquique y Alto Hospicio evidencian que se puede ser parte del fenómeno de la verticalización sin tener el volumen poblacional y espacial de una ciudad metropolitana.

En este sentido, ciertos aspectos del orden metropolitano, como los que se refieren a la tipología de los edificios de altura, que se desarrollaron para resolver problemas espaciales y residenciales, demuestran ser desplazables, sin la necesidad de movilizar con ellos a la realidad metropolitana propiamente como tal.

Esta capacidad de desplazarse fuera de su marco de origen y de reproducir fuera de él sus modos de orden de los elementos de su entorno es propio de las tecnologías, independiente de su tamaño, composición material o función. Este aspecto de los edificios de altura refuerza su sentido técnico original (la forma sigue a la función) y reafirma a la verticalización, su efecto conjunto, como un fenómeno que implica un régimen de orden urbano, en este caso, metropolitano.

El alto grado de estandarización de las formas, las materialidades, las instalaciones y los programas involucrados en el diseño y en el funcionamiento de los edificios de altura, es un reflejo de los diversos órdenes sociotécnicos consolidados en ellos y, a su vez, aseguran que tales modos de orden de las cosas se repitan en cada uno, configurando un orden residencial y urbano particular, enactado cada vez que se está frente a un edificio. 
Como se seńalaba al principio de este texto, tanto Hilberseimer (1999) como Le Corbusier (1985), prefiguraron un régimen urbano basado en los edificios de altura, así como William Levitt lo hizo sobre la base de la casa prefabricada en los Estados Unidos de la década de 1950. Sin embargo, la clave de esto no está ni en el edifico ni en la casa, sino en la estandarización del espacio residencial y en su disposición a ser replicado, como lo remarca la casa Farnsworth de Mies van der Rohe, también parte de la misma década.

La verticalización no es sólo un fenómeno que ocurre con la proliferación de los edificios residenciales de altura, en este caso, en las ciudades chilenas. Es, sobre todo, un régimen de orden de lleva a que las prácticas comerciales, materiales, arquitectónicas y residenciales den ese resultado. Y deviene en esto cuando sus modos de ordenar los elementos, que componen normativamente el habitar en el espacio residencial, alcanzan un grado de legitimidad tal que nos parecen invisibles.

El que la verticalización se constituya como un régimen urbano de orden metropolitano y se desplace hacia ciudades que no lo son resulta interesante, sobre todo porque ello podría presentarla como un indicador de la metropolitización de las ciudades intermedias o como un indicador de que las prácticas de edificación y residencialidad, en ciudades de menor tamaño, han asumido el estándar de las metrópolis, haciéndose parte de su ecología simbólica y cultural.

Esta segunda opción abre la posibilidad de entender lo urbano (y/o lo metropolitano) a partir de la operación de sus regímenes de orden y no solo desde la métrica de la densidad poblacional o de la extensión territorial. Esto permite problematizar las ciudades a partir de sus formas de ordenar el mundo y planificarlas desde el sentido de sus operaciones, poniendo su densidad o su tamaño dentro de una función que incluya sus formas, sus colores y otros ordenes distinguibles a simple vista, pero que no estén narrados como los órdenes urbanos en la actualidad.

\section{Referencias}

Blečić, I., y Cecchini, A. (2017). On the Antifragility of Cities and of their Buildings. City, Territory and Architecture, 4(3), 1-11. https://doi.org/10.1186/s40410-016-0059-4

Boltanski L., y Thévenot L. (2006). On Justification. Economies of Worth. Nueva Jersey: Princeton University Press.

Bowker, G., y Star, S. L. (1999). Sorting Things Out. Classification and Its Consequences. Cambridge: The MIT Press.

Goldthorpe, J. (2017). La sociología como ciencia de la población. Madrid: Alianza Editorial.

Graham, S. (2016). Vertical: The City from Satellites to Bunkers. Nueva York: Verso.

Grubbauer, M. (2010). The High-Rise Office Tower as a Global 'Type': Exploring the Architectural World of Getty lmages and Co. En M. Guggenheim y O. Söderström (eds.), Re-shaping Cities. How Global Mobility Transforms Architecture and Urban Form (pp. 63-80). Londres: Routledge.

Guggenheim, M. (2009). Building Memory: Architecture, Networks and Users. Memory Studies, 2(1), 39-53.

https://doi.org/10.1177/1750698008097394

Hilberseimer, L. (1999). La arquitectura de la gran ciudad. Barcelona: Editorial Gustavo Gili.

Hommels, A. (2005). Studying Obduracy in the City: Toward a Productive Fusion between 
Technology Studies and Urban Studies. Science, Technology, \& Human Values, 30(3), 323-351. https://doi.org/10.1177/0162243904271759

Ingold, T. (2010). The Textility of Making. Cambridge Journal of Economics, 34(1), 91-102. http://dx.doi.org/10.1093/cje/bep042

Instituto Nacional de Estadísticas (INE). (2006). Manual para el Análisis del Formulario Único de Edificación. Santiago de Chile: INE.

Instituto Nacional de Estadísticas (INE). (2007a). Definición y listado de ciudades de Chile. Santiago de Chile: INE. Recuperado de http://observatoriourbano.minvu.cl/indurb/wp_ pre_ciudades.asp?idComciu $=0$

Instituto Nacional de Estadísticas (INE). (2007b). Metodología Encuesta de Edificación Mensual. Santiago de Chile: INE.

Latour, B. (1987). Science in Action: How to Follow Scientists and Engineers Through Society. Cambridge: Harvard University Press.

Latour, B. (2001). La esperanza de Pandora. Ensayos sobre la realidad de los estudios de la ciencia. Barcelona: Gedisa.

Law, J. (2015). What's Wrong with a One-World World? Distinktion: Journal of Social Theory, 16(1), 126-139. http://dx.doi.org/10.1080/16 00910X.2015.1020066

Law, J., y Mol, A. (2009). El actor-actuado: La oveja de la Cumbria en 2001. Política y Sociedad, 45(3), 75-92. Recuperado de http://revistas.ucm.es/index.php/ POSO/article/view/ POSO0808330075A
Law, J., y Singleton, V. (2013). ANT and Politics: Working in and on the World. Qualitative Sociology, 36(4), 485-502. https://doi.org/10.1007/ s11133-013-9263-7

Le Corbusier. (1985). La ciudad del futuro. Buenos Aires: Ediciones Infinito.

Mahoney, J. (2015). Process Tracing and Historical Explanation. Security Studies, 24(2), 200218. http://dx.doi.org/10.1080/09636412.20 15.1036610

Marín, H., Ruiz-Tagle, J., López-Morales, E., Orozco, H., y Monsalves, S. (2019). Gentrificación, clase y capital cultural: transformaciones económicas y socioculturales en barrios pericentrales de Santiago de Chile. Revista Española de Investigaciones Sociológicas, (166), 107-134. http://dx.doi.org/10.5477/cis/reis.166.107

Martínez, J. P., y López, E. (2018). Massive Housing in Central Neighborhoods: Who Is Responsible for the Excessive Densification? ARQ (Santiago), (98), 144-153. https://dx.doi. org/10.4067/S0717-69962018000100144

Ramos, C. (2018) Dispositivo de evaluación y gubernamentalidad del sistema educacional: entretejimiento de ciencia social y poder. Cinta moebio, (61), 41-55. http://dx.doi.org/10.4067/ S0717-554X2018000100041

Star, S. L., y Griesemer, J. R. (1989). Institutional Ecology, 'Translations' and Boundary Objects: Amateurs and Professionals in Berkeley's Museum of Vertebrate Zoology, 1907-39. Social Studies of Science, 19(3), 387-420. https://doi. org/10.1177/030631289019003001 
Thévenot, L. (2016). La acción en plural. Una introducción a la sociología pragmática. Buenos Aires: Siglo XXI.

Thompson, J. (2001). El escándalo político: poder y visibilidad en la era de los medios de comunicación. Barcelona: Paidós
Vergara Vidal, J. (2017). Verticalización. La edificación en altura en la Región Metropolitana de Santiago (1990-2014). Revista INVI, 32(90), 9-49. http://dx.doi.org/10.4067/ S0718- 83582017000200009

\footnotetext{
"Torre de 21 pisos en Rancagua sin ascensores devela problema en mantención de edificios", en El Mercurio, 6 de abril de 2017.

"Providencia identifica 150 edificios donde se ejerce la prostitución", en La Tercera, 4 de abril de 2017; y "Matthei contra las prostitutas: "Iremos edificio por edificio para erradicar esta práctica'”, en El Mostrador, 5 de abril de 2017.

"Más de 500 viviendas por edificio: Las cifras detrás de los criticados 'guetos verticales'", en Emol, recuperado de http://www.emol.com/noticias/Nacional/2017/04/12/853908/Mas-de-500-viviendas-por-edificio-Los-numeros-de-los-criticados-guetos-verticales.html

4 Francisco Sabatini: “'Guetos Verticales': ¿Densidad o discriminación?”, en El Mostrador, 28 de julio de 2017; “Torres de Estación Central abren debate sobre la calidad de vida en viviendas de clase media", en El Mercurio, 07 de abril de 2017; y “'Guetos verticales', Ias infraviviendas de Santiago desatan polémica social”, en EFE, recuperado de https://www.efe.com/efe/cono-sur/cronicas/guetos-verticales-las-infraviviendas-de-santiago-desatan-polemica-social/50000803-3242081

5 "Guetos Verticales?". Carta del arquitecto Yves Besancon, en El Mercurio, 20 de abril de 2017 y “¿Guetos verticales, intendente?”. Carta de Paulina Henoch, Libertad y Desarrollo, en El Mercurio, 19 de abril de 2017.

6 "Ex contralor critica concepto de 'guetos verticales', pero recalca sus beneficios", en Emol, recuperado de http://www.emol.com/noticias/Nacional/2017/04/15/854315/ Ex-contralor-defiende-guetos-verticales-y-recalca-beneficios-de-cuestionada-construccion.html

7 "'Guetos verticales': Intendente Metropolitano pide a inmobiliarias que se autorregulen a la hora de construir", en Emol, recuperado de http://www.emol.com/noticias/Nacional/2017/04/11/853675/Guetos-verticales-Intendente-Metropolitano-pide-a-inmobiliarias-que-se-autorregulen-a-la-hora-de-construir.html. "Dirigente de la CChC y edificación de 'guetos verticales': La ética es de cada uno", en Emol, recuperado de http://www.emol.com/noticias/Economia/2017/04/11/853699/Dirigente-de-la-CChCy-guetos-verticales-Hay-una-debil-regulacion-y-una-alta-demanda.html. "Tras polémica por 'guetos verticales', inmobiliarias definen marco de autorregulación", en El Mercurio, 16 de agosto de 2017.
} 